\title{
Politique
}

\section{La présence du Québec en Amérique latine}

\section{Daniel Gay}

Numéro 7, hiver 1985

Projection internationale du Québec

URI : https://id.erudit.org/iderudit/040477ar

DOI : https://doi.org/10.7202/040477ar

Aller au sommaire du numéro

Éditeur(s)

Société québécoise de science politique

ISSN

0711-608X (imprimé)

1918-6584 (numérique)

Découvrir la revue

Citer cet article

Gay, D. (1985). La présence du Québec en Amérique latine. Politique, (7), 33-52. https://doi.org/10.7202/040477ar d'utilisation que vous pouvez consulter en ligne.

https://apropos.erudit.org/fr/usagers/politique-dutilisation/ 


\title{
La présence du Québec en Amérique latine
}

\author{
Daniel Gay \\ Université Laval
}

La récente réaffirmation par l'État québécois de la quête d'un destin international pour le Québec et les efforts considérables consentis en vue de le doter d'une politique internationale, fournissent l'occasion de réfléchir sur la présence du Québec dans le monde et plus particulièrement en Amérique latine (et dans les Caraïbes). En effet, de toutes les régions du Tiers-monde, l'Amérique latine est celle qui, pour différentes raisons - que nous ne pourrons qu'effleurer plus loin -, a toujours, au moins dès le début du $\mathrm{XIX}^{\mathrm{e}}$ siècle, fasciné l'imagination des clercs; c'est aussi le lieu où, au fil des ans, la présence du Québec s'est affirmée le plus, semble-t-il, surtout depuis la fin de la seconde guerre mondiale.

Entreprendre le bilan de cette présence n'est pas une tâche facile: il n'existe pas une tradition de recherche dans ce domaine. Le présent essai se ressent donc de certains tâtonnements. Notre ambition est plutôt modeste: d'une part, susciter des pistes de recherche, notamment dans la perspective de l'analyse historique et celle de la sociologie politique des relations internationales du Québec; d'autre part, contribuer à alimenter la discussion sur le thème important des relations Québec-Amérique latine. 
En premier lieu, nous procédons à une brève esquisse historique de l'intérêt exprimé par des membres des élites québécoises pour l'Amérique latine et des premières manifestations de la présence du Québec dans cette région. Nous tentons, ensuite, de caractériser celle-ci au niveau de la prédominance de ses modalités actuelles. Enfin, cet essai débouchera sur quelques réflexions en rapport avec la qualité de cette présence.

\section{De l'intérêt historique à la présence actuelle du Québec}

On peut identifier provisoirement trois grandes étapes dans le développement de l'appropriation symbolique de l'Amérique latine, au Québec, chacune d'elles recouvrant des tendances diverses.

La première étape (1842-1860) est celle de la découverte initiale, facilitée par les dépêches internationales et quelques études spécialisées en provenance de l'étranger, de quelques pays d'Amérique latine, de la part de rares penseurs engagés dans la quête de modèles de développement politique et social pour le Québec. Pour certains, ces modèles sont positifs: ainsi, l'histoire, le code civil, l'organisation socioéconomique et le système politique du Paraguay et de l'Uruguay sont-ils l'objet d'un examen attentif et bénéficient-ils d'une évaluation enthousiaste de la part de Maximilien Bibaud, président de l'Institut Polytechnique du Canada (Bibaud, 1857). Il en est de même du Mexique de la Réforme libérale qui captive l'imagination d'un Geoffrion, de l'Institut canadien (Costisella, 1968: 143-148). Ces auteurs expriment aussi une sympathie sans bornes pour les peuples en lutte et se réjouissent des déboires des puissances coloniales. Par exemple, Bibaud (1848: I-VII, et 1852: 50-51) plaide vigoureusement pour «la sauvegarde des petites puissances", en se référant au Mexique, au Pérou, au Paraguay et à Haïti. Par contre, si les observations sur le Chili rapportées par Bolduc (1843), inaugure l'ère des «recherches sur le terrain», l'auteur n'exprime aucune sympathie pour les Chiliens 
qu'il trouve, par exemple, "généralement très laids» (50-52) ou "presque sans goût pour les belles lettres, les sciences et les arts" (91-94) et chez qui «on ne voit aucune manufacture, et tout vient encore des nations étrangères» (50). Aucun rapprochement entre le Québec et le Chili n'est possible.

La seconde étape (1860-1920) est caractérisée par trois tendances distinctes, la première prolongeant la «découverte sur le terrain" de l'Amérique latine. Ainsi, à la connaissance du Chili fondée sur l'expérience, sans doute partielle et biaisée, s'ajoute successivement celle du Mexique, d'un grand nombre de pays d'Amérique centrale, d'Amérique du Sud et de la plupart des Antilles (Abbé Montminy, 1888; abbé Provencher, 1890; abbé Poulin, 1921: voyages effectués en 1901; et Lippé, 1907). Les observations de ces auteurs, à l'exception de celles du Père Montminy - plus pragmatique dans son évaluation «des richesses inépuisables» des Antilles ainsi qu'à leurs «denrées qui sont devenues un besoin pour tous les peuples» (187-190) — sont marquées au coin d'un néo-ultramontanisme, d'un ethnocentrisme et, dans le cas surtout de Provencher et de Lippé, d'un racisme virulents. Néanmoins, parlant au nom des «Canadiens français (...) abandonnés à euxmêmes parmi une race ennemie», Provencher (150-257), découvrant qu'à Trinidad aussi, alors ex-colonie française, «il se faisait des efforts soutenus pour angliciser le peuple de ces colonies anglaises, et surtout de faire disparaître la langue française», félicite "ceux qui résistent autant qu'ils le peuvent à ce mouvement». Il se rend compte également que «le catholicisme n'est pas seulement canadien français» et que le «joual» québécois présente d'étonnantes analogies avec le créole martiniquais. Enfin, au niveau de la quête de modèles de développement, l'auteur note que «sous plus d'un chapitre, nous pourrions aller demander à cette colonie de nègres des exemples de sage administration", principalement au niveau de l'organisation des «collèges pour l'éducation supérieure, qui ne se limitent pas 
aux études classiques» comme au Québec. Malgré tout, tous les auteurs mentionnés affirment que l'Amérique latine ne peut avantageusement soutenir la comparaison avec "notre Canada» sous le rapport de la liberté, de la sécurité, de la paix, de la dévotion ou même de la moralité publique. Pour Lippé (180), les Mexicains sont une «race quêteuse». Selon Poulin, au contact de l'irreligion et de la vénalité qui sévit au Sud, le Québécois perdrait son âme. Naguère, Bolduc (180) se plaignait qu'au Chili, «les femmes sont (...) outrageusement décolletées».

La seconde étape est caractérisée aussi par des suggestions à l'effet que les pays du Tiers-monde de l'époque constituent, pour quelques-uns, un tremplin pour l'affirmation exceptionnelle de l'identité et le rayonnement à l'extérieur, sous l'égide de la «mère-patrie». Nous ne pouvons pas nous attarder ici sur les témoignages de Québécois qui, entre 1863 et 1890, participèrent aux guerres coloniales de la France en Algérie ou au Maroc, ou à celles de l'Empire britannique au Mozambique, en Rhodésie ou en Afrique du Sud. Rappelons seulement comment l'un d'eux perçoit la participation de son groupe à l'expédition française au Mexique. Notant que «nos ancêtres (qui ne manquaient pas une certaine gloire militaire (...) (mais qui n'étaient) plus qu'un humble pékin (sic), dans toute l'acception du mot (...)»(1874: 10-11), Faucher de Saint-Maurice avoue, en référence à cette participation, que c'était pour que «le Canada français réalisant ses destinées (se lève) devant les peuples latins étonnés» (1889: 222).

Troisième tendance: par contre, et surtout au tout début du $\mathrm{XX}^{\mathrm{e}}$ siècle, dans le contexte d'un nationalisme pan-canadien opposé aux ambitions de l'Empire britannique, certains pays d'Amérique latine sont perçus comme pouvant éventuellement former avec le Canada un bloc pour faire contrepoids à l'influence anglaise. Henri Bourassa (1916; 1916b), par exemple, manifeste à cet égard un intérêt soutenu pour l'Argentine catholique et 
francophile, et dont la nouvelle bourgeoisie se sent également menacée dans sa sécurité, surtout par l'action des marchands anglais, alors principaux importateurs de viande et de céréales. On retrouve ici les germes de l'idée de la «troisième force» qui serait éventuellement constituée du Canada et de certains pays d'Amérique du Sud, et celle du rôle d'intermédiaire que le Québec pourrait jouer entre «les Anglais» et les «Latins d'Amérique» - idées qui seront reprises et amplifiées dans certains milieux, surtout entre 1950 et 1960.

Dans la troisième étape (1920-1960), et surtout entre 1922 et 1950 , on assiste tant au niveau de l'écriture que de l'action à une véritable explosion de l'intérêt manifesté envers l'Amérique latine. Trois principales tendances s'y dessinent. La première est marquée par l'hégémonie de la doctrine de la «latinité», défendue dès 1922 surtout par l'Action française, et amplifiée par le mouvement ultranationaliste connu sous le nom de «L'Union des Latins d'Amérique», fondée en 1940. Essentiellement, selon l'étude qu'Iris S. Podea (1948) a consacrée à cette tendance, l'on assigne au Canadien français une identité latine, fondamentalement différente de celle des Canadiens anglais, des Britanniques, des Américains... Or, ceux qui habitent au Sud du Rio Grande sont aussi latins. C'est sur cette base que doit donc s'effectuer le «nécessaire rapprochement» entre Latins du Canada et Latins d'Amérique. Leur mission: sauver la civilisation menacée par les «barbares» que sont les Anglo-Saxons, les Juifs, les Allemands et les Asiatiques. Le destin du Québec est d'assumer le leadership de cette croisade (sur ce point, voir aussi Bruchési, 1951; Mgr. Maurault, 1947: 201; 211).

Cependant, cette idéologie du rapprochement n'est pas unanime. En effet, tout en partageant cet objectif, des penseurs comme Jean-Charles Harvey, du Jour, ainsi que des porte-parole du Bloc Populaire le fondent plutôt sur des considérations d'ordre 
géographique, politique ou culturel, qui n'ont rien à voir avec la doctrine de la latinité. Pour d'autres, l'établissement des liens entre le Québec et l'Amérique latine pourrait se réaliser aussi bien dans le cadre d'un "État français» que dans celui de la Fédération canadienne. Par ailleurs, selon certains groupes, toute décision relative à l'opportunité ou non d'un tel rapprochement devrait être plutôt dictée par des considérations de rentabilité économique: "En matière de commerce et de finances, il faut mettre de côté les considérations trop sentimentales et s'en tenir aussi rigoureusement que possible à la réalité objective» (Patry, 1945: «Avant-propos»). Enfin, si, pour des adeptes de la Latinité, le Québec doit entretenir des relations «en particulier avec les Sud-Américains qui ont avec nous des affinités de race et de culture» (Maurault, op. cit.: 245), certains groupes d'intellectuels inaugurent plutôt le rapprochement du Québec avec «les Amériques noires». Et, à l'approche de la Révolution tranquille, une partie de la jeunesse intellectuelle et ouvrière, qui accueillera avec enthousiasme Fidel Castro, en 1959, à Montréal - comme elle y applaudira également Patrice Lumumba, le nouveau Chef de l'exCongo belge - sera, elle, mue par d'autres préoccupations comme celles des luttes de libération des peuples...

Deux dernières caractéristiques importantes de la période 1920-1960. D'une part, l'élargissement et l'intensification de l'«action latino-américaine» au Québec: la multiplication des Clubs d'amitié (Canado-haïtienne, Québécoise-brésilienne...); l'engouement pour l'étude de l'espagnol, sous l'égide de l'Association Canada-Inter-Amérique de Montréal, du Cercle Cervantès de Québec...; l'accueil des premiers étudiants latino-américains à l'Université Laval et à l'Université de Montréal, dès la fin de la seconde guerre mondiale... D'autre part, pour la première fois, semble-t-il, l'État québécois manifeste, en 1943, son intention d'établir des agences en Amérique du Sud: le Ministre du Commerce de l'époque annonce même que des fonds seront prévus à cette 
fin dans le prochain budget - projet qui ne se réalisera pas toutefois, par crainte d'une confrontation avec Ottawa (Podea, 1948: 343). Néanmoins, en 1945, toujours selon Podea, «le Trésorier et Ministre de l'Industrie et du Commerce fait une visite officielle à Mexico et en Haïti» (Ibid.) Enfin, en septembre 1949, sous le gouvernement de Maurice Duplessis, l'État québécois est officiellement représenté à l'Exposition internationale de Port-auPrince en commémoration du bi-centenaire de la fondation de la Capitale d'Haïti.

Ainsi, multiple et contradictoire, l'histoire posait les jalons...

La présence actuelle du Québec

Par «présence du Québec», nous entendons généralement l'ensemble des pratiques (interventions de divers types; exécution de projets de développement; campagne d'évangélisation; activités économiques et socio-culturelles...) dans lesquelles sont engagés, dans un ou plusieurs pays d'Amérique latine, des ressortissants du Québec, au nom de l'État québécois ou de l'État fédéral canadien, ou à titre de représentants de collectivités non-gouvernementales (églises, $\mathrm{ONG}$, associations à vocation commerciale ou financière...). C'est la présence institutionnelle du Québec.

Certains types d'acteurs invisibles (entreprises multinationales, «filières québécoise» de la Mafia, services d'espionnage...), que les théoriciens des relations internationales ont tendance à ignorer, devraient être inclus dans cette catégorie.

On peut prendre également en considération l'action de groupes d'individus ou d'individus non-affiliés (e.g., les touristes, certaines «missions de bonne volonté ou d'amitié», les résidents québécois en Amérique latine») pour souligner la présence noninstitutionnelle du Québec. 
Enfin, il conviendrait d'analyser aussi la présence symbolique du Québec en Amérique latine. On se réfere ici aux effets qu'entraînent ou que sont susceptibles d'entraîner, au niveau de la constitution d'une «image internationale» du Québec, parmi les élites ou parmi des secteurs importants des populations latinoaméricaines, des interventions en rapport avec l'Amérique latine, de la part de l'État ou de collectivités non-gouvernementales (intellectuels, syndicats, groupes populaires, media...), ici même au Québec.

Nous proposons d'identifier quatre intervenants principaux, à savoir l'État québécois, les gens d'affaires, les missionnaires et les coopérants. Nous nous attarderons davantage sur les deux premiers.

1. L'État québécois. Jusqu'au début des années soixantedix, l'Église catholique et un certain nombre d'églises protestantes, un certain nombre de familles (à Buenos-Aires, à la Barbade, à Port-au-Prince, à São Paulo...), quelques commerçants, de nombreux coopérants québécois - ainsi que l'importante "Mission semi-officielle de Bonne Volonté, de Bonne Entente ou d'Amitié» (formée d'hommes d'affaires, de professionnels et d'universitaires) qui visita, en 1946, l'Argentine, la Bolivie, le Brésil, la Colombie, Cuba, l'Équateur, le Mexique, le Pérou, l'Uruguay et le Vénézuela (Maurault, 1947: 25-29) - constituèrent l'essentiel de la présence québécoise en Amérique latine.

Bien que la loi de 1939 autorise déjà l'ouverture d'agences gouvernementales en dehors du territoire (MAG, 1971-1972: 31), et en dépit de l'intérêt croissant manifesté par des représentants de gouvernements d'Amérique latine (Porto Rico, Chili, Colombie, Mexique et Argentine) en visite officielle au Québec, surtout entre 1971 et 1973, c'est seulement en 1978 que le gouvernement amorce une politique vis-à-vis de l'Amérique latine. Sans doute, 
en octobre 1974, le Québec signait-il une entente avec le Consejo nacional de investigaciones cientificas y técnicas d'Argentine, laquelle sera mise en œuvre en 1975-1976; de même se faisait-il représenter, cette même année, par un fonctionnaire québécois auprès de l'École de pêcherie de Colombie. Par ailleurs, un Conseiller du Ministère de l'Immigration représente le Québec en Haïti (septembre 1976), avec pour principale mission de s'occuper de l'émigration d'Haïtiens vers le Québec. C'est à ces trois événements qu'étaient limitées jusqu'ici les relations officielles du Québec avec l'Amérique latine, l'essentiel des activités internationales de celui-là se déroulant aux États-Unis (MAG, 1975-76: 55, et 1976-77: 21). Déjà, en 197273 , le rapport annuel du Ministère des affaires intergouvernementales, alors chargé de la conduite des relations internationales du Québec, avouait que "priorité fut accordée au commerce et à la coopération plutôt qu'à l'aide et au développement international, ce qui entraîne plus d'échanges avec les pays développés qu'avec les pays du Tiers-monde. Les disponibilités du trésor québécois ne lui permettaient pas d'agir autrement, d'autant plus que les citoyens du Québec contribuent déjà par leurs taxes à financer le budget d'aide canadien" (44) — en particulier, par l'intermédiaire de l'Agence canadienne de développement international (ACDI) et sans compter l'action non négligeable des associations volontaires et du «prosélytisme des missionnaires» (Ibid.).

Bien que l'État québécois, en tant que tel, soit alors surtout préoccupé par la francophonie internationale, il faut signaler que c'est en 1974 que l'Hydro-Québec réalise sa première percée en Amérique latine, à la faveur des contacts établis au Brésil, cette année, par la première mission effectuée en ce pays par le Ministère canadien de l'Industrie et du Commerce. Ainsi l'Hydro-Québec signe un contrat de $3000000 \$$ avec la société d'État Electrobras pour l'aménagement de la rivière Parana, à la frontière du Paraguay, et l'Institut de recherches en énergie du Québec (IREQ), qui 
relève d'elle, conclut également une entente de $800000 \$$ pour la conception d'un laboratoire de recherche au Brésil (Leroux, 1974: F-1).

Dans l'ensemble, les relations internationales du Québec se situent toujours dans le cadre de la politique étrangère du Canada. Toutefois, il est intéressant de noter qu'en 1978, le Conseil des ministres pose les jalons d' «une politique québécoise vis-à-vis de l'Amérique latine" — sans trop la définir. En tout état de cause, «au cours de l'année 1979-1980, le Québec s'est tourné résolument vers l'Amérique latine (...)» (MAG, 1979-1980: 44). Voici quelques faits qui illustrent à peine l'ampleur de l'intérêt nouveau et de la présence du Québec dans les pays du Sud. 1978: création, pour la première fois, d'une Direction des affaires d'Amérique latine au Ministère des Affaires intergouvernementales, et ouverture d'un dossier «Mexique»... 1979: ouverture d'une délégation du Québec en Haïti; mise en relation de la télévision haïtienne avec Radio-Québec; perfectionnement au Québec du personnel de l'École d'hôtellerie de Port-au-Prince; mandat confié à SOQUIP pour effectuer, à la demande du gouvernement haïtien, une étude globale sur le potentiel pétrolier d'Haïti; contrat de consultant et de gérance obtenu par Hydro-Québec International...; ouverture de la Délégation du Québec au Vénézuela... 1980: ouverture de la Délégation générale du Québec au Mexique; discussions entre l'Hydro-Québec et la Comisión Federal de Electricidad du Mexique en vue de concrétiser des projets de coopération; aide à la formation de cadres costaricains dans le domaine de l'administration de la justice, et à l'instauration d'un système d'informatique à l'Univesité du Costa Rica; contrat important obtenu par Hydro-Québec International, conjointement avec un consortium québécois, pour la mise en place, à Caracas, de lignes de transmission de haute tension...

Cette liste est forcément incomplète. Malheureusement, selon les informations puisées au Ministère des relations internationales, 
les Rapports annuels pour 1981-1982 n'étaient pas encore publiés. Néanmoins, celui de 1980-1981 (57-60) comporte un grand nombre d'informations sur des discussions alors en cours entre le Québec et des pays comme le Brésil, le Costa Rica, la république Dominicaine et la Colombie, en vue de l'élargissement de la présence du Québec dans plusieurs autres domaines d'activité. Mentionnons aussi l'action, sous forme d'aide ou d'appui consenti par le Québec dans le cadre d'opérations multilatérales encadrées, par exemple, par la Confédération latino-américaine des coopératives d'épargne et de crédit pour la mise sur pied, à Panama, d'un Centre de formation des dirigeants de coopératives de 18 pays regroupés par COLAC, action entreprise notamment avec la Société de développement international Desjardins; par l'Institut centre-américain d'administration publique, avec l'aide de l'ENAP pour l'établissement d'un programme de maîtrise devant répondre aux besoins des six pays centre-américains; par l'Association brésilienne des professeurs de français; par le Conseil latino-américain des sciences sociales, en y appuyant le Groupe de recherche sur l'Amérique latine...

En somme, récemment et jusqu'à 1984, «l'accent de l'action gouvernementale s'est déplacé vers la coopération à retombées technologiques, scientifiques et économiques. (...) Largement africaine et francophone dans les années 60, la relation du Québec avec le Tiers-monde s'est graduellement élargie. (...) Dans les pays en développement et les pays nouvellement industrialisés, le Québec agit surtout en Afrique du Nord, en Afrique de l'Ouest et en Amérique latine» (Landry, 1984). Haïti, au titre de la plus importante communauté francophone d'Amérique; le Vénézuela, qui se situe au carrefour des trois Amériques et premier producteur de pétrole dans la région; et le Mexique, en raison de sa proximité géographique et du potentiel d'échanges et de coopération qu'il représente - sont identifiés par l'État comme "premiers pied-àterre du Québec» en Amérique latine (MAG, 1979-1980: 45). 
2. Les gens d'affaires. On a déjà fait état de certaines des activités commerciales, en Amérique latine, de l'État québécois, par le biais de ses organismes para-publics comme Hydro-Québec, IREQ et SOQUIP. Quant aux entrepreneurs privés, qu'ils soient établis dans des pays d'Amérique latine ou soient engagés dans des relations commerciales de divers types avec ceux-ci, ils manifestent également la présence du Québec au Sud du Rio Grande. On ne peut qu'indiquer la nature de leurs activités et l'évolution globale de leur présence. Déjà, dès 1959, la société Torrington, de Bedford, dans les Cantons de l'Est, alors présidée par Guy Champagne, fait des affaires au Brésil où, jusqu'à 1974, elle investit plus de $1000000 \$$. De plus, cette année-là, elle se préparait à y investir encore $1250000 \$$ pour la construction d'une usine de fabrication de roulement à billes pour l'industrie automobile brésilienne. La Dominion Engineering Works, alors présidée par Max Drouin, associée au projet d'Itaipu de l'HydroQuébec (1974), s'occupait de la construction de turbines (Leroux, 1974: F-1).

En 1965, la Société Nationale des Antilles, de Ste-Marie de Beauce, est constituée afin de mettre en valeur les ressources de la plaine de Torbeck (dans le Sud d'Haïti) où elle avait déjà entrepris la construction d'une usine sucrière de $7000000 \$$ (Le Nouvelliste, 1965: 1). Quelques années plus tard (1979), l'exdirecteur du Conseil des ports nationaux de Québec se rendait en Haïti à titre de consultant pour la construction d'un nouveau port, alors que, la même année, à la suite d'une entente conclue par le Ministère québécois de l'Agriculture avec l'Association des abattoirs et la Fédération des producteurs de chair de poulet, celles-ci vendaient à la République de Cuba 11 millions de livres de chair de poulet (Le Soleil, 1975).

Du côté des institutions financières, la Banque canadienne nationale qui «apparaîtra vraiment comme une institution nationale 
et internationale, et non plus seulement québécoise», était dotée, en 1977, d'une filiale aux Bahamas (Le Devoir, 1977: 31).

Enfin, pour les années plus récentes (1980-1981), mentionnons les contacts importants conclus par la firme Bombardier (100 $000000 \$$ ) pour la construction de wagons du métro de Mexico, et par une douzaine de P.M.E. québécoises (8 $000000 \$$ ) en rapport avec le contrat signé par le gouvernement vénézuellien avec Hydro-Québec. Enfin, diverses entreprises, comme Forano et Géomins Ltée ainsi que d'autres firmes spécialisées (dans l'aménagement urbain, le traitement de déchets et le nettoyage des rues) s'établisssaient au Mexique (MAG, 1980-1981: 55). Soulignons aussi la présence, surtout dans les Caraibes, d'une autre catégorie de gens d'affaires: les spéculateurs fonciers.

3. Les missionnaires et les coopérants. Bien que dans l'écriture comptable de l'ACDI, les religieux et les religieuses qui combinent évangélisation et tâches de développement figurent comme des «coopérants» ou des ONG au même titre que des groupes laïcs engagés dans la coopération internationale, il convient de retenir la distinction plus ancienne entre missionnaires et coopérants, fondée sur l'activité principale de l'un ou de l'autre groupe.

Depuis la publication, plutôt apologétique et plus ou moins pertinente, de Lionel Groulx (1962), on ne possède pas encore une étude systématique sur la présence des missionnaires québécois en Amérique latine. Toutefois, présents dans la région depuis 1843 (en Équateur), mais en plus grand nombre surtout depuis les années cinquante, ils constituent, aujourd'hui, le plus important contingent de "missionaires canadiens» dans le monde, ces derniers étant concentrés davantage en Amérique latine: 1,409 ou 41\%, sur un total de 3,473 (Bureau de l'entr'aide missionnaire, 1983). Répartis, quoique de façon inégale, dans tous les pays de l'Amérique du Sud et dans la plupart des Antilles, ils s'occupent, les uns, 
d'éducation ou d'aide aux malades ou aux vieillards, les autres, d'organisation communautaire ou de conscientisation - en plus de leur tâche d'évangélisation.

Quant aux coopérants proprement dit, dont les organisations - comme SUCO, OXFAM-QUÉBEC, Carrefour Tiers-Monde, Développement et Paix, l'Association canadienne pour les Nationsunis, YMCA, l'OCSD... - s'occupent majoritairement ou en partie, de développement en Amérique latine, «ils se sont regroupés récemment en une Association québécoise des organismes de coopération internationale (AQOCI). Selon la présidente de l'ACDI, «les ONG ont passé globalement le cap de leur premier quart de siècle d'existence et, comme le montre certains sondages, près de $20 \%$ de la population contribue, en temps et en argent, à leurs activités» (Le Devoir, 1984: 4). Quelques-uns bénéficient également de l'appui financier du gouvernement québécois.

Voici quelques réflexions sur certains aspects touchant l'efficacité de la présence du Québec en Amérique latine.

Réflexions sur la qualité de la présence du Québec en Amérique latine

Bien que les ministères responsables des décisions relatives aux activités internationales du Québec ainsi que certains ONG se livrent, périodiquement à une autocritique interne de leur «mission», une évaluation critique, systématique et indépendante, de la présence institutionnelle et symbolique du Québec en Amérique latine, reste à faire. Elle paraît s'imposer d'abord au niveau de l'action de chacun des principaux groupes d'intervenants.

L'État publiait, en 1982, sous le titre Les délégations du Québec... une action concrète, un bilan de la valeur de ses exportations internationales, de 1978 à 1982, et selon 25 pays de destination, dont le Mexique, le Brésil, le Vénézuela et Cuba. Le tableau 1 
(19) révèle la croissance considérable de la valeur de ces exportations, que le Ministère des Affaires gouvernementales qualifie d'«investissements rentables pour le Québec, même quand on ramène leur action (celle «des conseillers économiques, culturels et touristiques, ou les spécialistes de l'immigration œuvrant tant au Québec qu'à l'extérieur ») à une simple question de dollars et de cents». De plus, lit-on encore dans le Rapport, «cette rentabilité va bien au-delà de ces seules considérations, puisque c'est la personnalité même du Québec qui s'affirme (...)»

Nonobstant cette appréciation triomphaliste, fondée sur une conception mercantiliste, de l'affirmation de la personnalité du Québec, celle-ci demeure problématique, compte tenu, par exemple, de la contradiction des orientations politiques, à l'égard de l'Amérique latine, des élites gouvernementales, et des problèmes liés à la philosophie et à la gestion de certains programmes de «coopération internationale». Ainsi, dans le premier cas, le premier ministre du Québec se prononce en faveur de l'Argentine dans la guerre qu'elle livre, en 1982, à l'Angleterre, pour la repossession des îles Malouines. Mais on apprend (Le Devoir, 19/06/82: 10) du Ministre des Transports, que l'État québécois avait loué à l'Argentine des avions ainsi que les services d'équipages québécois, pour aider ce pays à combattre des incendies en forêt, au coût de 1,3 million incluant un bénéfice net de $750000 \$$. Ce qui, dans l'estimation d'un lecteur du Devoir (26 juin 1982: 10) revient à «facturer l'Argentine avec $136 \%$ de profit net». D'autre part, alors que, le 29 mars 1980 (Le Devoir, 1980: 3), le Ministre de l'Immigration concélébrait à Montréal une messe à la mémoire de l'archevêque Romero, assassiné au Salvador, et qu'à l'instigation du gouvernement, l'Assemblée nationale du Québec condamnait à l'unanimité le régime de Duvalier, en Haïti (Haïti-Observateur, 1980: 6), "nous nous taisons lorsque des armes produites au Québec blessent et tuent au Nicaragua, au Guatémala, au Salvador» (Jurdant, 1984: 6; voir aussi Beauregard, 1984: 6). Enfin, des 
porte-parole du Comité Québécois d'Appui à la Résistance du Peuple Chilien protestaient, dans une lettre adressée au Ministre des Affaires intergouvernementales (De Serres et Michaud, 1979: 4), contre une décision du gouvernement consistant à permettre à la Société des Alcools du Québec d'offrir aux consommateurs québécois du vin en provenance du Chili et de l'Argentine, alors qu'il avait annoncé qu'il boycottait les vins d'Afrique du Sud.

Ce dont on peut être sûr, c'est qu'une société ne peut pas ruser longtemps avec ses principes. On ne peut plus parler de commerce sans tenir compte des droits fondamentaux des peuples. On ne peut pas se permettre d'être "progressiste» à temps partiel.

En ce qui a trait à l'évaluation de l'efficacité des programmes de coopération, on a le sentiment que ceux qui sont bien planifiés et qui répondent réellement aux attentes des «bénéficiaires", sont appréciés à leur juste valeur. La vérité est que, dans certains pays d'Amérique latine, les coopérants se substituent avantageusement à l'indifférence ou au cynisme des élites locales ou «nationales». Ils pénètrent parfois dans des régions désolées où l'État central n'est symbolisé, par les paysans, que par la venue périodique du policier ou du percepteur d'impôts. Il n'est donc pas étonnant que des coopérants, religieux ou laïques, soient «adoptés» par les habitants d'une localité.

Mais il y a des ombres au tableau. Certains coopérants sont incompétents; des «experts» recrutés en fonction du patronage ainsi que certains administrateurs de projets, parfois imposés aux professionnels du milieu, semblent ne penser qu'à s'amuser aux frais de la Princesse. Dans certains cas, leur recrutement est la récompense à leur loyauté politique. En examinant de près le récent Rapport de l'Institut Nord-Sud (1984) sur l'évaluation de trois projets d'envergure récemment réalisés ou en cours de réalisation en Haïti (Projet CRUDEM: formation professionnelle, Frères du Sacré-Cœur; Projet de la Faculté d'agronomie et de médecine 
vétérinaire, Université Laval, et Projet DRIP: développement rural intégré, Société d'expertise-conseil de Montréal, URBARCCanada Ltée), on relève des critiques du genre, à propos du projet du projet DRIP: «aucun système permettant une comptabilisation mensuelle ou annuelle d'engagements et de dépenses applicables aux diverses activités ou à l'ensemble du projet» (p. 98) ou encore: «Le volet administratif représente presque la moitié des décaissements, et plus de la moitié si l'on inclut l'élément gestion des activités sectorielles» (p. 107). Concernant le projet de l'Université Laval, le Rapport constate (p. 64) que, manquant de compétence en médecine tropicale et n'étant pas équipés pour résoudre le problème, les coopérants durent faire appel à des Français pour les seconder dans leurs tâches (p. 77). Enfin, nonobstant certaines évaluations positives du Projet CRUDEM, on note que «les Frères du Sacré-Coeur sont des pédagogues d'abord» et non pas des administrateurs et des gestionnaires. Vu leur «non compétence», ils durent céder la continuation du projet à la Banque mondiale, pour éviter la faillite (p. 81), même si, en accord avec le gouvernement haitien, les paysans qui participaient au projet (coopératives, banques d'épargne, centres d'enseignement en santé...) était payés 0,50 \$ par jour (Midy, 1974). Enfin, dans sa critique du Projet DRIP, le Rapport Nord-Sud constate que, si le programme d'alphabétisation a profité davantage aux pauvres, le programme de santé, par contre, était hors de portée des démunis. Quant à la construction des routes, elle a surtout favorisé les notables. Ainsi, «on peut douter de l'utilité du programme de construction de routes» (p. 133).

Dans les premières années de réalisation du Proejt CRUDEM (1968), un observateur autochtone se demandait déjà: "Présence canadienne et québécoise en Haïti : aide ou exploitation?» (Midy, 1973; voir aussi CASO et CHAP, 1972). La question est posée. 


\section{Conclusion}

Cette étude comporte des lacunes évidentes. Par exemple, nous n'avons pu montrer si, ou dans quelle mesure, certaines des tendances esquissées au début se prolongeaient aujourd'hui au niveau de l'expression de l'intérêt ou de la manifestation de la présence du Québec en Amérique latine. Il n'était pas possible non plus de relier les quelques données pertinentes à celle-ci aux transformations récentes de la société québécoise, dans le contexte du système international capitaliste. On aurait alors été fondé de poser les questions suivantes: en dépit du champ constitutionnel étroit dans lequel elle se meut, la présence "québécoise» est-elle réellement différente, au niveau de ses principes fondamentaux et de ses pratiques, de la présence "canadienne»? Quels seront les effets, au niveau de la politique internationale du Québec, du récent virage politique du gouvernement du Québec?

Cette étude se voulait seulement un premier essai de type monographique sur un sujet encore mal connu.

Cependant, un fait est certain. Lente à se manifester, la présence du Québec en Amérique latine aujourd'hui est une réalité. Multiple, elle est contradictoire aussi: elle paraît osciller entre l'humanisme et le souci de la rentabilité à tout prix. Enfin, expression d'une volonté d'affirmation et de rayonnement du Québec à l'extérieur, cette présence paraît problématique - en dépit de discours enthousiastes et généreux.

\section{Bibliographie}

BEAUREGARD, Chantal, «Le discours et les actes», Le Devoir, 25 avril 1984, p. 5. BIBAUD, Maximilien, Biographie des Sagamos illustres de l'Amérique septentrionale. Précédée d'un Index de l'bistoire fabuleuse de ce continent, Lowell, 1848.

BIBAUD, Maximilien, Résumé de l'entretien familier du président en ibef de l'Institut Polytechnique $d u$ Canada, à la réunion hebdomadaire du 30 avril 1852, l'an II de sa fondation, p. 44-55. 
BIBAUD, Maximilien, Deux pages de l'Histoire d'Amérique lues au cabinet de lecture, le 12 mai 1857, Montréal, Sénécal et Daniel, 1857.

BOLDUC, J. B. Z., Mission de Colombie, Québec, Imprimerie J. B. Fréchette, père, 1843.

BOURASSA, Henri, Hier, aujourd'bui, demain, Montréal, 1915.

BOURASSA, Henri, Independance or Imperial Partnership?, Montréal, 1915.

BRUCHÈSI, Le Canada français dans le monde, Montréal, La Société St-Jean-Baptiste, 1951.

Bureau de l'entraide missionnaire, Statistiques de la Confédération religieuse canadienne, 1983.

Comité haïtien d'action patriotique et Centre d'animation pour le service outre-mer, Dossier Haïti, Québec, 1972.

"Capital québécois en Haïti", Le Nouvelliste (Port-au-Prince), 4 février 1965, p. 1.

COSTISELLA, Joseph, L'esprit révolutionnaire dans la littérature canadienne-française (de 1837 à la fin de XIX' siècle), Montréal, Beauchemin, 1968.

DE SAINT-MAURICE, Faucher, De Québec à Mexico: souvenirs de voyage, de garnison, de combat et de bivouac, Montréal, Duvernay, 1874.

DE SAINT-MAURICE, Faucher, Notes pour servir à l'histoire de l'Empereur Maximilien du Mexique, Cité, Québec, 1889.

ENGLISH, Philip, L'Aide au développement du Canada à Haitti. Une étude indépendante, Institut Nord-Sud, Ottawa, 1984.

GROULX, Lionel, Le Canada français missionnaire: une autre grande aventure, Montréal, Fides, 1962.

JURDANT, Michel, "Le Nicaragua libre mais assiégé», Le Devoir, 9 août 1984, p. 7.

"La BCN intensifiera ses activités», Le Devoir, 9 décembre 1977, p. 1.

LANDRY, Bernard, «Le Québec dans le monde: entre la coopération et les relations commerciales", Le Devoir, 30 mai 1984, p. 9.

«Le capitaine Allard devient directeur du port», Le Soleil, 2 février 1975, p. C-13, Ministère des Affaires intergouvernementales, Les Délégations du Québec... une action concrète. Québec, 1982.

LEROUX, Roger, "La réputation du Québec en énergie électrique est un atout dans ses échanges avec le Brésil", Le Soleil, 6 novembre 1976, p. F-1.

LIPPÆ, J. A., Le tour du Mexique. Mon journal de voyage, Montréal, Arbour et Dupont, 1907.

MAUrault, Mgr. O., Par voies et par chemin de l'Air (Les Amériques), Les Éditions des Dix, Montréal, 1947.

MIDY, Franklin, «Présence canadienne et québécoise en Haïti; aide au exploitation?", Relations, 381, avril 1973, p. 102-107.

MIDY, Franklin, «Des œuvres charitables aux manœuvres politiques», Le Devoir, 4 mai 1974.

Ministère des Affaires gouvernementales, Rapports annuels pour les années 1967 à 1981 , Québec.

MONTMINY, abbé Th., De Québec aux Antilles. Notes de voyages, Québec, J. A. Langlais, 1890.

PATRY, André, «La République d'Haïti comme débouché pour le Canada», Mémoire présenté à la Faculté des Sciences sociales, Université Laval, 1945. 
PODEA, Iris S., "Pan American Sentiment in French Canada», International Journal, III4, Autumn 1948: 336-349.

POULIN, abbé A., Voyage de... Vol. I. À travers le Nouveau-Monde, imprimerie «Le Soleil", Québec, 1921.

PROVENCHER, Léon abbé, Une excursion aux pays tropicaux, Québec, Langlais, 1890. 